\title{
Nutritional effects on asthma aetiology and progression
}

\section{Patricia A Cassano}

That nutrition affects health would not be argued by many, but questions about specific strategies to prevent chronic disease onset or mitigate disease progression do not yield easy answers. The role of diet in lung health, including chronic obstructive pulmonary disease (COPD), asthma and pulmonary function, has been reviewed repeatedly, ${ }^{1-4}$ but whether accumulated findings represent causal links remains an open question. Continuing to build the evidence base is critical, and efforts in this direction will contribute to the formulation of well-informed public health programmes and policies to guide individual, group and industry-level decision making.

In this issue of Thorax, Allen et a ${ }^{5}$ report the results of a meta-analysis of observational epidemiological studies of vitamins $\mathrm{A}, \mathrm{C}$ and $\mathrm{E}$ in relation to asthma over the life course with the purpose of estimating the effect size for each association (see page 610). Their report summarises data from 40 publications, uses a guideline for reporting observational study meta-analyses $^{6}$ and provides a more comprehensive and rigorous approach than another recent overview $^{7}$ which concluded there is little or no evidence for a link between diet and asthma. Allen et a $t^{5}$ show that the dietary intake of vitamin $\mathrm{A}$ is lower in patients with asthma, and also lower in those with severe compared with mild disease. Lower intakes of vitamin $\mathrm{C}$ are associated with an increased risk of asthma-more specifically, there is a $12 \%$ increase in risk for low compared with high dietary vitamin C groups. Allen et a ${ }^{5}$ report little or no association of vitamin $\mathrm{E}$ with asthma risk, but studies were markedly more heterogeneous and therefore less amenable to summarisation. These findings, and other recent reports, continue to highlight an association between diet and asthma. If the associations are causal, then preventives and/or therapeutic targets would be identified; both are of critical public health importance in asthma.

Correspondence to: Dr P A Cassano, Division of Nutritional Sciences, Cornell University, Ithaca, NY 14850, USA; pac6@cornell.edu
What is the current state of the dietasthma hypothesis, and what can we take forward into the next generation of studies? In the -omic age, novel approaches to the study of asthma are likely to be transformative, yielding a new understanding of intermediate end points and phenotypes. Linking epidemiological studies to emerging biological models of asthma aetiology will improve the evidence for when and how diet plays a role. Indeed, studies of in utero dietary exposures are important to understanding the role of fetal imprinting ${ }^{8}$ in asthma risk and the role of diet in a developmental context. ${ }^{9}$ To the extent that disease arising in adulthood has its origins in early life, studies of adult diet may lead to erroneous answers. On the other hand, adult diet may indeed be a contributor to disease occurrence or reappearance through mechanisms such as antioxidant function ${ }^{10}$ or anti-inflammatory capacity, ${ }^{2}$ as supported by the findings of Allen et al. ${ }^{5}$ Ongoing challenges include questions about whole foods versus isolated nutrients, the measurement of nutrient status at the optimal time and in target tissues, an understanding of usual nutritional status and potential to benefit (thresholds, non-linear effects), the complex interrelations among dietary components and diet as a modifier of environmental exposures that contribute to risk. The heterogeneity in published studies may reflect variation in all of the above, or it may reflect variation in confounding or other systematic errors that, once resolved, will leave us with more complete answers to complex questions.

Is there a need for further randomised trials on diet and asthma? Past randomised controlled trials of the link between diet and disease have often yielded null and even paradoxical results in comparison with the observational epidemiological studies leading up to the trials. To date, published trials of nutritional interventions for asthma, COPD or pulmonary function are typically of short duration and conducted under specific conditions (environmental challenge) to address the question of diet as a therapeutic. The results from these trials are mixed. A recent meta-analysis of vitamin $\mathrm{C}$ does not support an effect on the amelioration of asthma symptoms, ${ }^{11}$ a large trial of vitamins $C$ and $E$ and beta-carotene found no effect of intervention on pulmonary function or hospitalisation rates for COPD or asthma, ${ }^{12}$ and the ATBC study reported no effect of vitamin $\mathrm{E}$ or betacarotene on respiratory symptom outcomes. ${ }^{13}$ As a firmer basis for causal hypotheses emerges, better designed trials will become possible. In the meantime, there is a continuing need for creative epidemiological solutions-for example, studies of diet hypotheses that use Mendelian randomisation ${ }^{14}$ - to continued exploration of the role of diet in asthma aetiology and progression.

Competing interests: None.

Thorax 2009;64:560. doi:10.1136/thx.2008.112011

\section{REFERENCES}

1. Weiss ST. Obesity: insight into the origins of asthma. Nat Immunol 2005;6:537-9

2. Litonjua AA. Dietary factors and the development of asthma. Immunol Allergy Clin North Am 2008;28:60329, ix.

3. McKeever TM, Britton J. Diet and asthma. Am J Respir Crit Care Med 2004;170:725-9.

4. Smit HA. Chronic obstructive pulmonary disease, asthma and protective effects of food intake: from hypothesis to evidence? Respir Res 2001;2:261-4.

5. Allen S, Britton JR, Leonardi-Bee JA. Association between antioxidant vitamins and asthma outcome measures: systematic review and meta-analysis. Thorax 2009;64:610-19.

6. Stroup DF, Berlin JA, Morton SC, et al. Metaanalysis of observational studies in epidemiology: a proposal for reporting. Meta-analysis Of Observational Studies in Epidemiology (MOOSE) group. JAMA 2000;283:2008-12.

7. Gao J, Gao X, Li W, et al. Observational studies on the effect of dietary antioxidants on asthma: a metaanalysis. Respirology 2008;13:528-36.

8. Miller RL. Prenatal maternal diet affects asthma risk in offspring. J Clin Invest 2008;118:3265-8.

9. Dietert RR, Zelikoff JT. Early-life environment, developmental immunotoxicology, and the risk of pediatric allergic disease including asthma. Birth Defects Res B Dev Reprod Toxicol 2008;83:547-60.

10. Kelly FJ. Vitamins and respiratory disease: antioxidant micronutrients in pulmonary health and disease. Proc Nutr Soc 2005;64:510-26.

11. Ram FS, Rowe BH, Kaur B. Vitamin C supplementation for asthma. Cochrane Database Syst Rev 2004;(3):CD000993.

12. Anon. MRC/BHF Heart Protection Study of antioxidant vitamin supplementation in 20,536 high-risk individuals: a randomised placebo-controlled trial. Lancet 2002;360:23-33.

13. Rautalahti M, Virtamo J, Haukka J, et al. The effect of alpha-tocopherol and beta-carotene supplementation on COPD symptoms. Am J Respir Crit Care Med 1997;156:1447-52.

14. Ebrahim S, Davey SG. Mendelian randomization: can genetic epidemiology help redress the failures of observational epidemiology? Hum Genet 2008;123:15-33. 